Conclusion The rapid increase of numbers and rates of multiples born to women over 30 years in Japan is mainly attributed to iatrogenic rather than spontaneous multiple births. The effect of non-ART fertility treatment is large recently.

\section{SP6-34 LAUGHTER AS A PREDICTOR OF SUBSEQUENT DISABILITY IN COMMUNITY-DWELLING ELDERLY IN JAPAN}

doi:10.1136/jech.2011.142976q.5

${ }^{1} \mathrm{M}$ Hirosaki, ${ }^{*}{ }^{2} \mathrm{Y}$ Ishimoto, ${ }^{2} \mathrm{Y}$ Kasahara, ${ }^{3} \mathrm{R}$ Sakamoto, ${ }^{2} \mathrm{M}$ Ishine, ${ }^{2} \mathrm{~T}$ Wada, ${ }^{3} \mathrm{~K}$ Okumiya, ${ }^{2} \mathrm{~K}$ Matsubayashi. ${ }^{1}$ Kansai University, Osaka, Japan; ${ }^{2}$ Kyoto University, Kyoto, Japan; ${ }^{3}$ Research Institute of Humanity and Nature, Kyoto, Japan

Introduction The importance of positive emotion to our health has been recognised and an increasing number of studies have examined the association between positive emotion and health. The value of experience laughter has been receiving attention recently. The aim of this study was to examine the association between frequency of laughter (FOL) and subsequent disability in community-dwelling elderly in Japan.

Methods A 1-year prospective study was conducted among 162 community-dwelling people aged 65 years and older who participated in annual health check-ups for the community's elderly and confirmed to have no disability in performing activities of daily living $(\mathrm{ADL})$ at baseline interview. Measures included functional ability, sociodemographic and psychological factors, medical conditions, and self-ratings of frequency of laughter (FOL). OR and 95\% CI of functional disability 1 year later in relation to the low-level of FOL vs higher levels of FOL were calculated using logistic regression models. Results Low-level FOL at baseline was significantly related to functional disability 1 year later in univariate analyses (OR 5.78, $95 \%$ CI 2.03 to 16.51). In multivariate analyses, low FOL was also significantly correlated with functional disability, even after controlling for sociodemographic variables, psychological factors, and medical conditions.

Conclusion Our findings suggest that low frequency of laughter is associated with functional decline in community-dwelling elderly in Japan.

\section{SP6-35 PSYCHOLOGICAL WELL-BEING AND OBESITY IN OLDER BRITISH ADULTS}

doi:10.1136/jech.2011.142976q.6

${ }^{1} \mathrm{~B}$ Stewart-Knox, ${ }^{*}{ }^{1} \mathrm{M}$ Duffy, ${ }^{1} \mathrm{~B}$ Bunting, ${ }^{1} \mathrm{H}$ Parr, ${ }^{2} \mathrm{M} \mathrm{D}$ V de Almeida, ${ }^{3} \mathrm{M}$ Gibney. ${ }^{1}$ University of UIster, Coleraine, UK; ${ }^{2}$ University of Porto, Portugal, Portugal; ${ }^{3}$ University College Dublin, Dublin, Ireland

The aim of this research was to better understand the psycho-social context of obesity to inform prevention and treatment of obesity at both the individual and public health level. A representative sample of middle-aged adults aged $40+$ years recruited in Great Britain (GB) $(\mathrm{N}=1182)$ underwent interview assisted survey to explore associations between waist circumference (WC), demographic factors, physical activity, dietary habits (FFO), life events (LES), Resilience (RS11), Mood (MS), Hopelessness (BDI) and Perceived Stress (PSS4). Data were analysed using multiple linear regression analysis for which predictors were entered simultaneously in four blocks: (1) demographic factors; (2) stressful life events; (3) diet/activity; and, (4) psychological measures. Continuous measures (age, years of education and psychometric scores) were adjusted to determine any interactions with sex. Being male, older, having spent fewer years in education, not working, being sedentary, having experienced more illness-related life events and lower scores on the resilience scale were all independent predictors of a larger WC. Intervention to promote physical activity and resilience may help to ameliorate midsection obesity, particularly among older males.

\section{SP6-36 FUNCTIONAL EDENTULISM AND THE NEED FOR TOTAL PROSTHESIS AMONG BRAZILIAN ELDERLY: MULTILEVEL EFFECTS}

doi:10.1136/jech.2011.142976q.7

${ }^{1} \mathrm{R}$ Moreira, ${ }^{*} \mathrm{~L}$ Barrozo, ${ }^{2} \mathrm{~J}$ Pereira. ${ }^{1}$ Oswaldo Cruz Foundation, Ministry of Health, Recife, Pernambuco, Brazil; ' Universidade de São Paulo, São Paulo, São Paulo, Brazil

Introduction The demographic and epidemiological transitions are causing changes in the health profile worldwide. Among the various areas of healthcare, oral health expressed is in a precarious situation. The objective was to identify the individual and contextual factors associated with tooth-loss in the elderly in Brazil.

Methods Analyses were based on the 2003 cross-sectional national epidemiological survey of the oral health of the Brazilian population, which covered 5349 individuals (age 65-74 years). Multistage cluster sampling was used. The dependent variable was functional edentulism and the need for total prosthesis. Independent variables were classified according to the individual or contextual level. A multilevel Poisson regression model was adopted.

Results Two analyses were carried out. In the first, living in rural areas and being female were associated with functional edentulism. In the second analysis, the contextual variables associated with reduced need for total prosthesis were: greater coverage of the first dental consult program (at regional level), average number of years of schooling (at state level) and population size. Living in rural areas, a greater number of individuals per room, having had the last dental consultation in the public sector, being male, non-white and of greater age were associated with the need for total prosthesis.

Conclusion The study revealed the key contextual and individual aspects associated with greater tooth-loss. The knowledge of the need for total prosthesis will be able to guide the work of those working in the area of Epidemiology of Oral Health.

\section{SP6-37 PREDICTING DENGUE FEVER INCIDENCE IN SELANGOR USING TIME SERIES ANALYSIS TECHNIOUE}

doi:10.1136/jech.2011.142976q.8

S A Shah, ${ }^{*}$ J A Sani. Department of Community Health, Faculty of Medicine, Universiti Kebangsaan Malaysia, Kuala Lumpur, Malaysia

Introduction The war against dengue fever-the most important vector borne disease in Malaysia, has yet to be won. Throughout the years, Selangor has been the champion, bearing about half of the total number of dengue fever cases in Malaysia. This study aims to develop forecasting models based on the number of dengue fever occurrence in 3 areas in Selangor, namely Sepang, Kuala Selangor and Shah Alam.

Methods Monthly data of dengue fever occurrence based on date of onset of dengue fever symptoms for Sepang, Kuala Selangor and Shah Alam from 2004 to 2007 were collected. Patterns of monthly dengue fever occurrence were used to develop suitable forecasting models. The most suitable models were then used to predict the dengue fever occurrence in 2008.

Results All 3 study locations showed overall increasing trend of dengue cases over the 4 years with seasonal pattern. Forecasting of dengue cases for 2008 was performed using the best model identified for each location, after seasonal factors were accounted for. All models identified for forecasting was found to be suitable as shown by the residual autocorrelation function (ACF) and partial autocorrelation function (PACF).

Conclusion Predicting dengue fever occurrence using time series analysis can be useful in the long term planning of dengue fever control and prevention programme. However, longer period of data are required to allow for validation of the forecasting models, which could not be performed in this study. 